\title{
A new in-gas-laser ionization and spectroscopy laboratory for off-line studies at KU Leuven
}

\author{
Yu. Kudryavtsev*, P. Creemers, R. Ferrer, C. Granados, L.P. Gaffney ${ }^{1}$, M. Huyse, E. Mogilevskiy ${ }^{2}$, \\ S. Raeder ${ }^{3}$, S. Sels, P. Van den Bergh, P. Van Duppen, A. Zadvornaya \\ KU Leuven, Instituut voor Kern- en Stralingsfysica, Celestijnenlaan 200D, B-3001 Leuven, Belgium
}

\section{A R T I C L E I N F O}

\section{Article history:}

Received 28 July 2015

Received in revised form 17 February 2016

Accepted 18 February 2016

Available online 4 March 2016

\section{Keywords:}

Resonance ionization spectroscopy

Laser ion source

Gas cell

Gas jet

De Laval nozzle

\begin{abstract}
A B S T R A C T
The in-gas laser ionization and spectroscopy (IGLIS) technique is used to produce and to investigate short-lived radioactive isotopes at on-line ion beam facilities. In this technique, the nuclear reaction products recoiling out of a thin target are thermalized and neutralized in a high-pressure noble gas, resonantly ionized by the laser beams in a two-step process, and then extracted from the ion source to be finally accelerated and mass separated. Resonant ionization of radioactive species in the supersonic gas jet ensures very high spectral resolution because of essential reduction of broadening mechanisms. To obtain the maximum efficiency and the best spectral resolution, properties of the supersonic jet and the laser beams must be optimized. To perform these studies a new off-line IGLIS laboratory, including a new high-repetition-rate laser system and a dedicated off-line mass separator, has been commissioned. In this article, the specifications of the different components necessary to achieve optimum conditions in laser-spectroscopy studies of radioactive beams using IGLIS are discussed and the results of simulations are presented.
\end{abstract}

(ㄷ) 2016 Elsevier B.V. All rights reserved.

\section{Introduction}

The in-gas laser ionization and spectroscopy (IGLIS) technique developed at KU Leuven has been used at the Leuven isotope separator on line (LISOL) facility to produce short-lived radioactive beams in different regions of the chart of nuclei using light and heavy-ion induced fusion or fission reactions [1-5]. In this technique, the nuclear reaction products recoiling out of a thin target are thermalized and neutralized in a high-pressure noble gas, resonantly ionized by the laser beams in a two-step process [6], and then extracted from the ion source to be finally accelerated and mass separated. In this way isobaric and isotopic selectivity can be achieved. High efficiency and selectivity of the ion source by a dual chamber approach [7] allows performing in-gas cell resonance ionization spectroscopy of exotic atoms. Using this method ground- and isomeric-state properties, such as isotope or isomer shifts, charge radii and nuclear magnetic moments of isotopes pro-

\footnotetext{
* Corresponding author. Tel.: +32 16328515.

E-mail address: Yuri.Kudryavtsev@fys.kuleuven.be (Yu. Kudryavtsev).

1 Present address: School of Engineering, University of the West of Scotland, Paisley PA1 2BE, United Kingdom.

2 Address: Faculty of Mechanics and Mathematics, Lomonosov Moscow State University, Leninskie Gory, 1, 119992 Moscow, Russia.

3 Present address: Helmholtz-Institut Mainz, 55128 Mainz, Germany.
}

duced in fusion evaporation reactions have been measured in copper [8], silver [9] and actinium. The achievable spectral resolution of the original method, whereby resonant laser ionization is performed inside the buffer gas cell, is limited by the collision broadening mechanism to values not better than $3-5 \mathrm{GHz}$. Implementation of resonance laser ionization in the supersonic gas jet overcomes this limitation and allows increasing the spectral resolution by more than one order of magnitude in comparison with in-gas-cell ionization spectroscopy [10-12]. In recent online experiments with radioactive actinium isotopes the spectral line width has been reduced down to $300 \mathrm{MHz}$ [13]. Further improvements can make it possible to obtain a resolution of the same order of magnitude as with other high-resolution laser photoionization technique, such as collinear resonance ionization spectroscopy (CRIS) [14,15]. To obtain the maximum efficiency and the best spectral resolution, properties of the supersonic jet and the laser light must be optimized [12]. To perform these studies a new off-line IGLIS laboratory, including a new highrepetition-rate laser system and a dedicated off-line mass separator, has been commissioned. In this article, the specifications of the different components necessary to achieve optimum conditions in laser-spectroscopy studies of radioactive beams using IGLIS will be discussed and the results of simulations will be presented. An optimized IGLIS setup to perform laser ionization 
spectroscopy including high repetition lasers will be installed at the focal plane of the Super Separator Spectrometer $\left(S^{3}\right)$ [16], which will be coupled to the superconducting linear accelerator of the SPIRAL2 facility at GANIL [17]. An international network that includes 14 research groups [18] with the common goal of implementing and/or developing the IGLIS technique is currently established to inform about their progress and coordinate the collaboration among them to further optimize the IGLIS method.

\section{General description}

A schematic layout of the laboratory is shown in Fig. 1. It is distributed in two rooms, being the first a temperature stabilized $\left( \pm 0.5^{\circ} \mathrm{C}\right)$ clean room (ISO8) with a Safety Access System (SAS). This room houses the laser system that provides several beams to perform high-resolution laser photo-ionization spectroscopy in a supersonic gas jet. The laser beams, indicated with $\lambda_{1}$ and $\lambda_{2}$ representing their respective wavelength, are sent to the front-end of the separator situated in the mass-separator room. The front-end hosts a gas cell coupled to a de Laval nozzle to create the gas jet [19]. The gas handling system provides purified noble gases into the gas cell. Laser produced ions are transported by radio frequency quadrupole (RFQ) ion guides up to an extraction electrode. Subsequently, ions are focused by an electrostatic Einzel lens and are sent towards a dipole magnet. The frontend of the separator is at ground potential, but the separator itself is placed on a high voltage $(-40 \mathrm{kV})$ platform. Before and after the dipole magnet the ion beam is monitored in beam diagnostic chambers which include Faraday cups and a beam profile meter based on multichannel plates (MCP) and phosphor screens.

\subsection{In-gas jet laser ionization spectroscopy: general principle}

The basic principle of the in-gas jet laser ionization consists in the resonance excitation of seeded atoms to an intermediate atomic level with subsequent ionization of excited atoms via continuum- or autoionizing states. A de Laval nozzle is used to create an axisymmetric supersonic gas jet. This gas jet is characterized by a low atom density and a low temperature, which result in a strong reduction of the collision- and Doppler broadening mechanisms in comparison with those existing in the gas cell. Ionization takes place in the volume defined by the overlap of the two laser beams with the jet. To obtain optimum spectral resolution as well as to avoid ionization inside the gas cell, a cross laser beam interaction with the gas jet has to be used, as pointed out in [12]. The main parameter that characterizes the property of the jet is the Mach number $(M)$. Fig. 2 shows the contribution of the Doppler broadening to the spectral line width $(438 \mathrm{~nm})$ for actinium atoms and the diameter of the jet as a function of the Mach number. At a Mach number 12 the jet diameter is $10.5 \mathrm{~mm}$ and the spectral resolution is limited to $80 \mathrm{MHz}$. To achieve this resolution, the laser system must operate with a suitable laser bandwidth and the ion guide system should be able to accept the ion beam formed at the interaction region with the required diameter.

\subsection{Laser system}

Two step ionization schemes are frequently used for selective ionization of atoms in the gas cell and in the supersonic gas jet [6]. The laser system consists of two Nd:YAG INNOSLAB lasers (EdgeWave $\mathrm{GmbH}$ ) with a maximum repetition rate of $15 \mathrm{kHz}$ that pump two dye lasers and a dye amplifier, see Fig. 1. The average output power of each pump laser is $90 \mathrm{~W}$ with a frequency doubling module at $532 \mathrm{~nm}$ (green) or $36 \mathrm{~W}$ with a frequency tripling module at $355 \mathrm{~nm}$ (UV) and a pulse length of 8-10 ns. The radiation of the Nd:YAG laser 1 is split to pump the first step dye laser and the pulsed dye amplifier. The radiation of the Nd:YAG laser 2 is used only to pump the second step dye laser. Both lasers are time-synchronized with a jitter better than $3 \mathrm{~ns}$.

Dye lasers (CREDO, Sirah Lasertechnik $\mathrm{GmbH}$ ) cover the tuning range of the fundamental wavelength from 375 to $900 \mathrm{~nm}$. Both dye lasers are equipped with a grating lift option that allows changing between two gratings of 1800 lines $/ \mathrm{mm}$ and 3000 lines $/ \mathrm{mm}$ inside the laser cavity to be used with the green or the UV pump wavelengths, resulting in a laser bandwidth of $0.08 \mathrm{~cm}^{-1}$ and $0.05 \mathrm{~cm}^{-1}$, respectively. Additionally, the bandwidths can be increased by a factor of four by changing an intracavity prism expander. This option can be useful to search for unknown atomic transitions of rare isotopes in on-line experiments. Frequency conversion units (FCU) in both dye lasers allow frequency doubling of the fundamental wavelength insuring an continuous tuning range of the laser system from $215 \mathrm{~nm}$ up to $900 \mathrm{~nm}$. The laser pulse width of fundamental and second harmonic laser beams is 7-9 ns.

To perform high-resolution spectroscopy in the supersonic gas jet, laser radiation with a bandwidth smaller than the residual Doppler broadening is required. This light is obtained by seeding of a single mode continuous wave (CW) tunable laser beam in a pulsed dye amplifier. The narrow bandwidth $(1 \mathrm{MHz}$ at $5 \mu$ s and $654.8 \mathrm{~nm}$ ) laser light from the TA Pro (TOPTICA Photonics AG) Master Oscillator Power Amplifier (MOPA) design is focused into a one- or two stage dye amplifier. The amplified pulsed radiation is then frequency doubled in a frequency conversion unit to get a wavelength of $\lambda_{1}=327.4 \mathrm{~nm}$ that can be used for the first step excitation of copper atoms. The spectral line profile, obtained as the Fourier transform of the pulse time profile, has a full width at half maximum (FWHM) of $63 \mathrm{MHz}$ for a laser pulse length of $7 \mathrm{~ns}$. The spectrum of the CW laser is monitored by a scanning Fabry-Perot interferometer (SFPI) with a free spectral range (FSR) of $4 \mathrm{GHz}$ and finesse better than 400 . Fundamental wavelengths of the dye lasers and the amplifier are measured in sequence by a wavelength meter HF-ANGSTROM WS/7 (HighFinesse GmbH) with an absolute accuracy of $60 \mathrm{MHz}$. The wavelength meter has an internal calibration option and additionally can be externally calibrated by a frequency stabilized $\mathrm{He} / \mathrm{Ne}$ laser. The laser bandwidth of the dye lasers is measured by a homemade air-spaced Fabry-Perot Interferometer (FPI). The time synchronization of the laser beams that are sent towards the separator is controlled by fast photodiodes.

The diameter of the gas jet is defined by the Mach number of the nozzle, see Fig. 2. The saturation energy fluence needed for the first excitation step [12] can be easily provided by the present laser system for Mach number lower than 15. The saturation fluence of the second ionization step is about $0.4 \mathrm{~mJ} / \mathrm{cm}^{2}$ for an autoionization cross section of $1 \cdot 10^{-15} \mathrm{~cm}^{2}$. The argon jet velocity is $560 \mathrm{~m} / \mathrm{s}$ and to ensure interaction of all atoms with lasers operated at $10 \mathrm{kHz}$ pulse repetition rate the size of the laser beam along the jet axis ( $\lambda_{2}$ in Fig. 3) should be at least $5.6 \mathrm{~cm} \mathrm{[12].} \mathrm{The} \mathrm{jet}$ diameter with $M=7$ is about $0.5 \mathrm{~cm}$. To provide complete spatial overlapping and saturation of the second step, a pulse energy of $1.2 \mathrm{~mJ}$ is required for a laser with a beam are of $0.5 \mathrm{~cm} \times 5.6 \mathrm{~cm} \approx 3 \mathrm{~cm}^{2}$. A way of reducing the required pulse energy would be to use a multipath laser beam $(0.5 \mathrm{~cm}$ in diameter) arrangement, which consists of two mirrors at a small angle along the gas jet.

A fraction of both laser beams is deflected by beam splitters towards an atomic beam unit (ABU). Here, laser ionization of the same atoms as those seeded in the gas jet is carried out in a $90^{\circ}$ cross-beam geometry that provides a spectral resolution closed to the laser bandwidth. The laser-produced ions are mass separated in a time of flight mass spectrometer (TOF MS). The atomic beam is produced by resistive heating of a graphite crucible and 


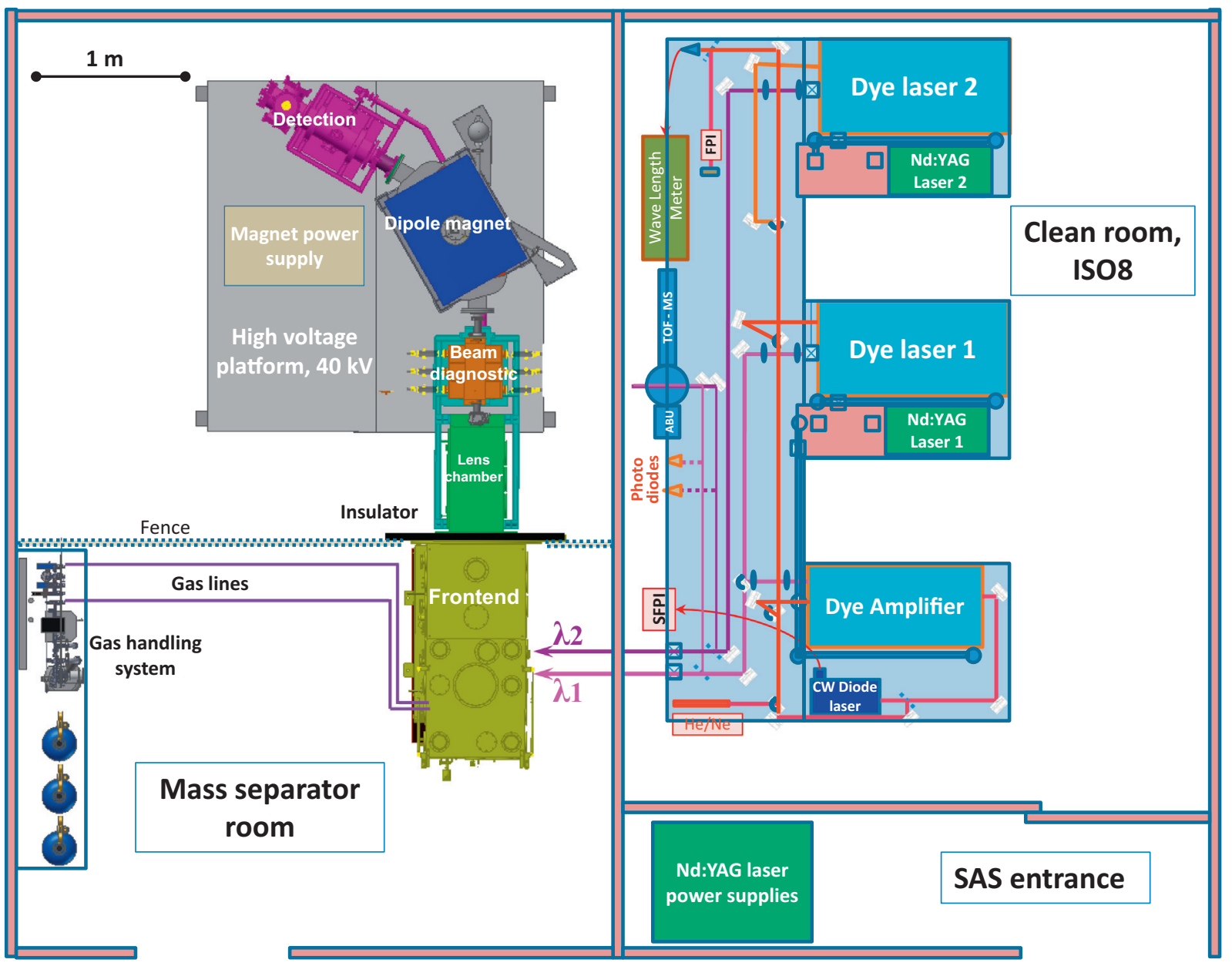

Fig. 1. Schematic layout of the IGLIS laboratory at KU Leuven.

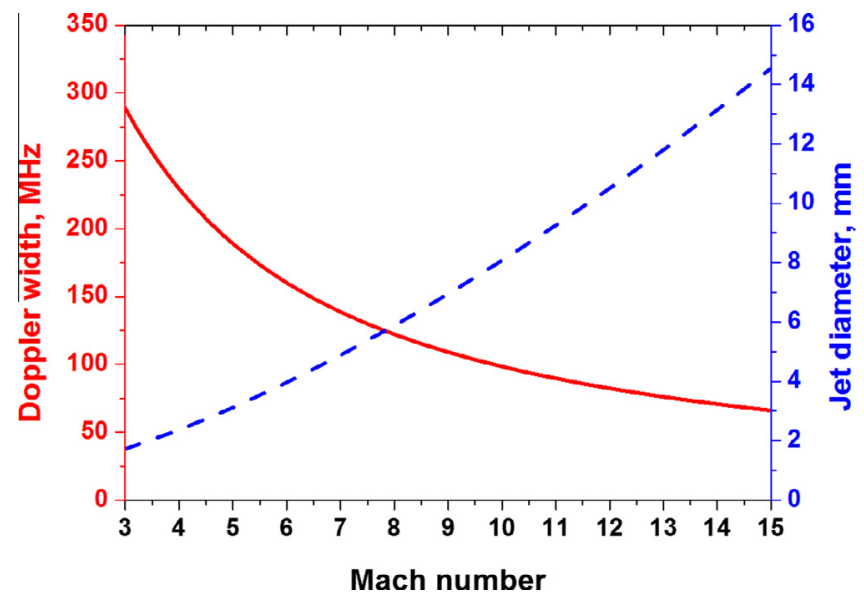

Fig. 2. Contribution of the Doppler broadening to the spectral line width for the $438 \mathrm{~nm}$ transition in actinium (solid line) and the diameter of the jet (dashed line), produced by a de Laval nozzle with a throat diameter of $1 \mathrm{~mm}$, as a function of the nozzle's Mach number.

collimated through a set of diaphragms. Laser ionization takes place in the first stage of a dual-stage ion extraction system where ions are accelerated to $1.2 \mathrm{keV}$, subsequently mass separated in a field-free region of $0.6 \mathrm{~m}$ long and detected in an MCP detector. A mass resolving power $(\mathrm{m} / \delta \mathrm{m})$ of 80 is obtained that allows separating two stable isotopes of copper $\left({ }^{63,65} \mathrm{Cu}\right)$ and acquiring ion signals from both isotopes at the same time.

\subsection{Mass separator frontend}

The mass separator frontend consists of three chambers, see Fig. 3. In the gas cell chamber the supersonic gas jet is formed by a de Laval nozzle. Atoms that are seeded inside the gas cell from a resistively-heated filament are ionized in the gas jet by two laser beams $\lambda_{1}, \lambda_{2}$. The laser produced ions are transported by an $S$ shaped RFQ (S-RFQ) ion guide towards the differential pumping chamber 2, where they are guided first by a differential pumping RFQ (DP-RFQ) used as a pumping barrier and further by a larger diameter linear RFQ towards chamber 3. Two stages of differential pumping ensure a vacuum sufficient to extract ions and accelerate them up to $40 \mathrm{keV}$ for those jets with Mach number greater than 4.5 at a stagnation pressure in the gas cell of 500 mbar.

\subsubsection{Gas cell chamber}

The gas cell chamber hosts the gas cell, the S-RFQ, some optical elements to shape the laser beams and numerous observation windows, pumping ports and feedthroughs (electrical, gas inlet, etc.). In order to be able to adjust the distance between the cell and the S-RFQ for the different Mach number jets the gas cell is placed on a movable platform while the S-RFQ is fixed. Special conditions have to be considered to form a long and uniform gas jet. The flow uniformity is strongly influenced by the background pressure $P_{1}$, which should be ideally equal to the static flow pressure $P$ defined as 


\section{TOP VIEW}
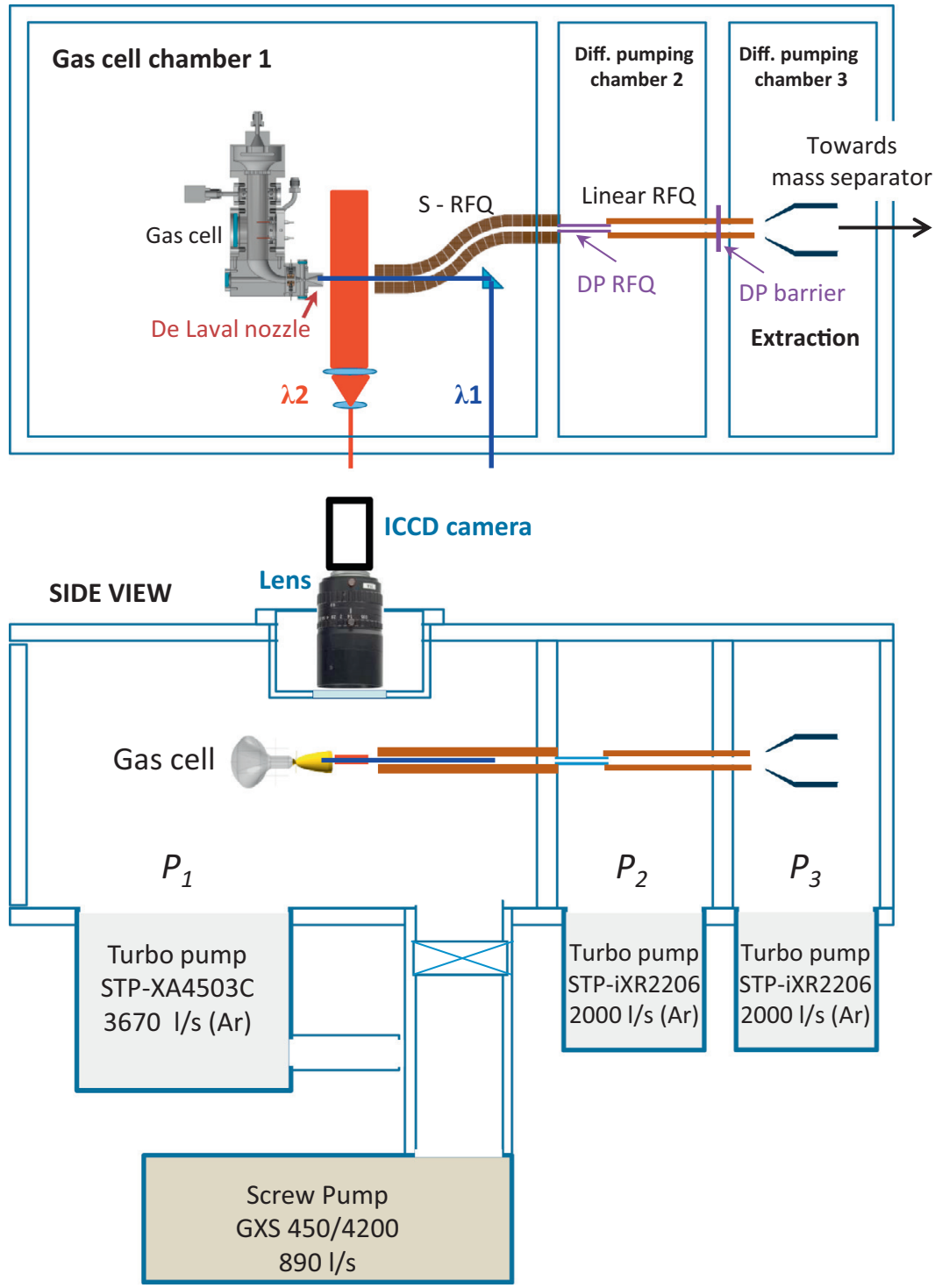

Fig. 3. Top- and side-view layout of the mass separator frontend. See text for details.

$\frac{P}{P_{0}}=\left[1+\left(\frac{\gamma-1}{2}\right) M^{2}\right]^{\frac{-\gamma}{\gamma-1}}$

where $P_{0}$ is the stagnation pressure in the gas cell, $\gamma=C_{p} / C_{v}$ is the ratio of specific heat capacities, which for monatomic gases like argon and helium equals to $5 / 3$, and $M$ is the jet Mach number. Thus, the pressure in the gas cell chamber $P_{1}$ is defined by the pressure in the gas cell and by the jet Mach number. This dependence is shown in Fig. 4 for three values of the stagnation pressure $P_{0}$. As can be seen a wide pressure range has to be provided by the pumping system. The higher the Mach number the lower the background pressure $P_{1}$ should be in the gas cell chamber. To ensure this pressure range two modes of operation for vacuum pumps are foreseen (see the side view in Fig. 3). For relatively small Mach numbers only a screw pump GXS 450/4200 (Edwardsvacuum), $890 \mathrm{l} / \mathrm{s}$ is used while for larger Mach numbers a turbo pump STP-XA4503C (Edwardsvacuum), $3670 \mathrm{l} / \mathrm{s}$ is operated with the GXS 450/4200 as a backing pump. Matching the background pressure with that of the jet at given Mach number ensures collimation of the de Laval jet expansion and uniform flow conditions at the longest distance

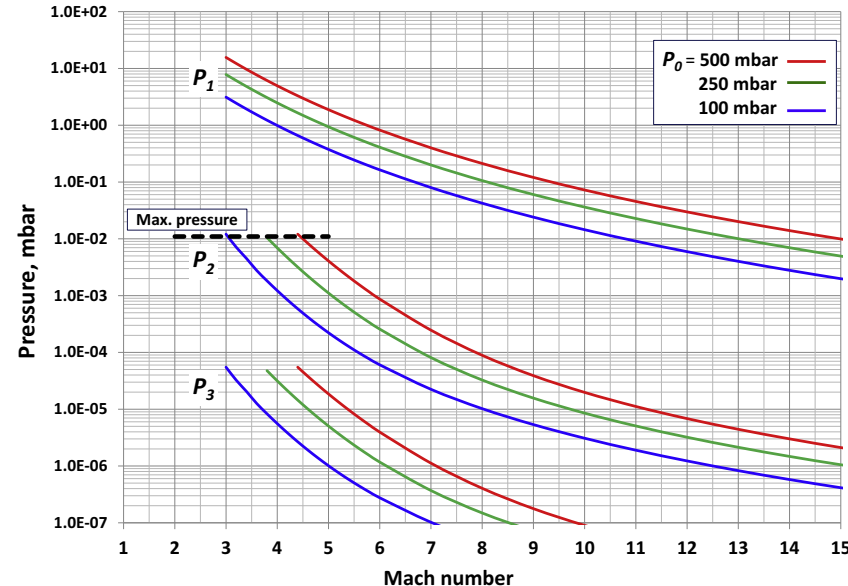

Fig. 4. Required background pressure $P_{1}$ in chamber 1 , and the pressures $P_{2}$ and $P_{3}$ obtained by differential pumping respectively in chamber 2 and in chamber 3 for different pressures $P_{0}$ in the gas cell, as a function of the nozzle's Mach number. 
$[19,20]$. The pressure $P_{1}$ can be adjusted by changing the rotation speed of the pumps.

Differential pumping between the chambers is provided by two turbo pumps STP-iXR2206 (Edwardsvacuum) $2000 \mathrm{l} / \mathrm{s}$ and by the DP RFQ and the DP barrier (see Fig. 3 top view). The DP RFQ, a cylindrical wedge-type RFQ [21], has a length of $11 \mathrm{~cm}$ and an effective inner cross section of $0.8 \mathrm{~cm}^{2}$. The area of the DP barrier is $1 \mathrm{~cm}^{2}$. The pressures in chamber $2\left(P_{2}\right)$ and in the chamber 3 $\left(P_{3}\right)$ are shown in Fig. 4 as a function of the Mach number as well. While calculating the flow conductance of the DP RFQ the viscous-, intermediate- and molecular character of the gas evacuation was taken into account. Pressure suppression between chambers 1 and 2 of 4000 can be achieved in the case of molecular regime, which is valid at high Mach numbers, and of only 300 for a viscous flow present at low Mach numbers. Since the maximum working pressure of the turbo pump is limited to $10^{-2} \mathrm{mbar}$, the system has a low limit for the Mach number 4.5 at $P_{0}=500$ mbar. This limit is indicated by a dashed line in Fig. 4. The reachable pressure in the chamber 3 is well below $10^{-4}$ mbar for all Mach numbers.

The turbo pump and the screw pump have different working pressures and different maximum pumping speeds. Fig. 5 shows Mach numbers that can be reached as a function of the stagnation argon pressure $P_{0}$ for two modes of operation. In the screw pump mode (solid lines) the achievable Mach number depends only on the throat diameter of the nozzle $d$ and is almost insensitive to $P_{0}$. Only at low pressures the Mach number decreases because of the pumping speed reduction of the screw pump at low pressures. At $P_{0}=500$ mbar and $d=1 \mathrm{~mm}$ the maximum Mach number equals to 9.5. In case of turbo pump mode (dashed lines) higher Mach numbers can be obtained in comparison with the screw pump because of the higher pumping speed of the turbo pump. However, there is a limitation for the maximum allowable argon flow through the pump of $6.63 \mathrm{~Pa} \mathrm{~m}^{3} / \mathrm{s}$. This value corresponds to the maximum inlet argon pressure of $1.4 \cdot 10^{-2}$ mbar. This restricts the maximum stagnation pressure in the gas cell. At $P_{0}=500 \mathrm{mbar}$ the maximum throat diameter equals to $0.7 \mathrm{~mm}$. The dotted line connects points with maximum pressures that can be obtained for different throat diameters. The reduction of the Mach number with $P_{0}$ for each throat is related to the reduction of the pumping speed at higher pressures.

\subsubsection{Gas cell and gas handling system}

The gas cell has been designed as a prototype for the on-line gas cell that will be used at the rare elements in-gas laser ion source (REGLIS) of the $S^{3}$ facility, GANIL (France) [16]. A cut view of the cell is shown in Fig. 6a. The dual chamber concept consisting in separation of the stopping- and the laser ionization regions [7] is kept in this design as for searching for unknown atomic transitions, low-resolution spectroscopy in the gas cell is needed. In the REGLIS gas cell the ion beam from $S^{3}$, of about $4 \mathrm{~cm}$ in diameter, will enter the cell through a thin foil window and will be ideally stopped in the center of the cell. This part of the cell has the shape of a cylinder with an oval-shaped cross section to house the window and to reduce the total volume of the cell and consequently the evacuation time of the radioactive isotopes. In the off-line cell the ion beam entrance window is replaced by a fused silica viewport. On the opposite side of the viewport two filaments are placed in a water-cooled CF flange that can be resistively heated to produce by evaporation a continuous source of atoms and thus imitate the behavior of the radioactive atoms thermalized in the buffer gas during on-line experiments. The filaments can also be irradiated by a laser beam to ablate of a burst of atoms to monitor the gas cell evacuation time.

The design of the cell has been optimized by means of gas flow simulations using the COMSOL software package [22]. This optimization deals with minimizing the diffusion losses and the

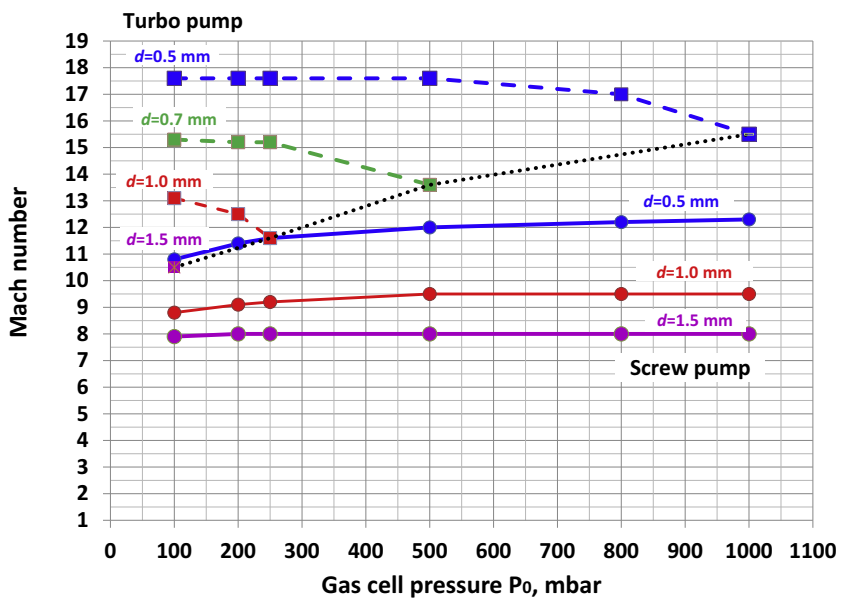

Fig. 5. Ultimate Mach number as a function of the stagnation argon pressure $P_{0}$ in the gas cell for two modes of operation: the screw pump mode (solid lines) and the turbo pump mode (dashed line). In the later the screw pump is used as a backing pump. The dotted line connects points with maximum allowable pressures by the turbo pump.

transport time of the atoms towards the nozzle. The calculated flow pattern is show in Fig. 6b. The purified gas from the gashandling system is fed into the cell by an inlet tube of $4 \mathrm{~mm}$ in diameter. An inlet gas velocity of about $10 \mathrm{~m} / \mathrm{s}$ results from a volumetric argon flow rate of $140 \mathrm{~cm}^{3} / \mathrm{s}$ evacuated through the exit throat with a diameter of $1 \mathrm{~mm}$. Once the buffer gas atoms leave the inlet tube, their velocity is reduced by collisions with an obstacle plate. A uniform and laminar flow is subsequently generated inside the cell by a flow straightener, which consists of 51 tubes (nine rows with five tubes and two rows with three tubes) of $3 \mathrm{~mm}$ in diameter and $5 \mathrm{~mm}$ long. The gas pressure is measured by a pressure transducer (Omega Engineering limited) in the range $0-2000$ mbar. Beyond the central part of the gas cell where the radioactive atoms are stopped, the oval-shaped cross section of the cell is smoothly changed with the $90^{\circ}$ elbow to a round section of $10 \mathrm{~mm}$ in diameter at the position of an ion collector. The collector consists of two semicircular electrodes with a small gap between them. During on-line conditions it collects nonneutralized ions from the stopping region. Downstream of the ion collector the diameter is gradually reduced to $6 \mathrm{~mm}$. In this volume the in-gas cell laser ionization takes place if both laser beams are sent transversally. On the other hand the properties of the ion collector can be tested by creating laser ions along the longitudinal laser beam path. The de Laval nozzles can be connected to the cell. The convergent part of these nozzles has an entrance diameter of $6 \mathrm{~mm}$.

The main parameter of the gas cell is the evacuation efficiency, which is determined by the diffusion losses of the seeded atoms into the cell walls during their transport from the point of origin to the cell exit. The losses are defined by a diffusion coefficient of atoms in the carrier gas, by the gas cell pressure, by the dimensions of the cell and by the exit throat diameter. In the evaluation of the cell efficiency, the incoming source of atoms was modeled as a small sphere ( $1 \mathrm{~mm}$ radius) with a steady atom creation rate. The evacuation efficiency is defined as the ratio of outgoing flux through the throat of the nozzle to the incoming flux of atoms. The efficiency was calculated for a cell width $(w)$ of 20,30 and $40 \mathrm{~mm}$ ( $y$-direction, Fig. 6a) and different initial source positions inside the cell. The evacuation efficiency for the different initial atom source positions and different pressures in the $40 \mathrm{~mm}$ gas cell are shown in Fig. 7a for the throat diameter of $1 \mathrm{~mm}$. The efficiency curves exhibit an asymmetric shape relative to the 

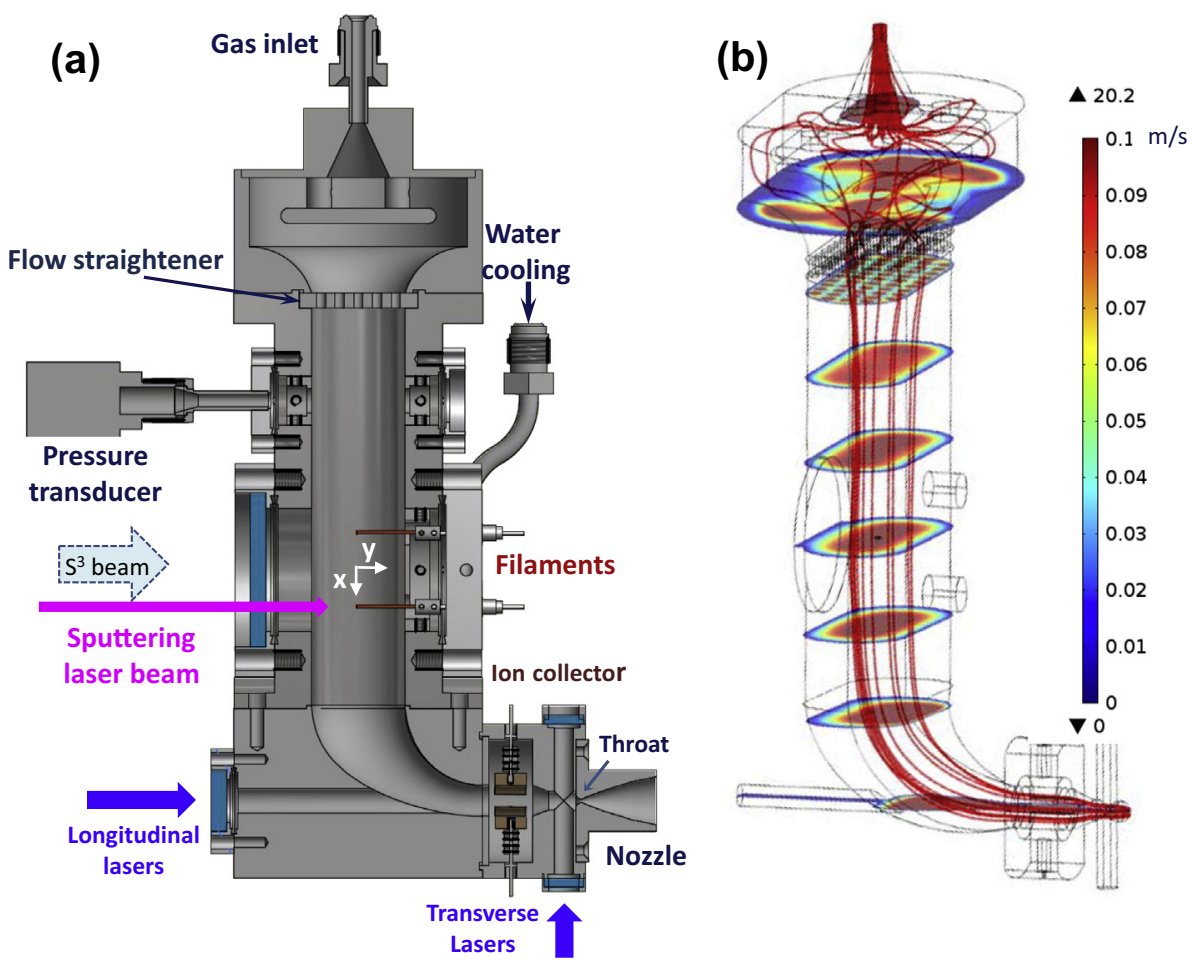

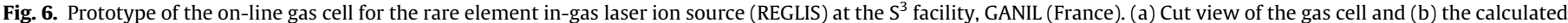
gas flow pattern. The laser beam trajectories are given for in-gas cell ionization.

central plane $(x=0)$ of the cell being slightly higher for those atoms that are closer to the inner surface of the elbow $(y>0)$. The efficiency is noticeably reduced for a gas pressure of 100 mbar in comparison with 500 mbar. This is related to the fact that the diffusion coefficient is inversely proportional to the gas pressure. The coefficient was taken to be $3.8 \cdot 10^{-5} \mathrm{~m}^{2} / \mathrm{s}$, which is relevant for atoms with mass $A=100$ in argon at 500 mbar. Regions close to the walls $(y= \pm 18 \mathrm{~mm})$ show a strongly reduced efficiency, while the change of coordinates along the cell axis $(x= \pm 18 \mathrm{~mm})$ is not significant for high pressures. Since the evacuation time depends on the throat diameter, the efficiency is reduced for smaller throat diameters because of bigger diffusion losses.

The evacuation time was also calculated for different initial positions of atoms in the $40 \mathrm{~mm}, 30 \mathrm{~mm}$ and $20 \mathrm{~mm}$ cells. In Fig. $7 \mathrm{~b}$, it is shown for the $40 \mathrm{~mm}$ cell for atoms from the $y z$ plane with $x=0$. The evacuation properties of the $30 \mathrm{~mm}$ and $20 \mathrm{~mm}$ cells have been investigated for a simulated distribution of stopped ${ }^{254}$ No beam from $S^{3}$. The initial spatial distribution was simulated by the SRIM program [23]. The thickness of the entrance window was adjusted to stop species in the center of the cell and the spread of the ${ }^{254}$ No beam was found to be of $7 \mathrm{~mm}$ (90\% of the ions). The calculated average evacuation time for atoms from the stopping region is about $630 \mathrm{~ms}$ for the $30 \mathrm{~mm}$ cell. This time is reduced down to about $400 \mathrm{~ms}$ for the $20 \mathrm{~mm}$ cell. Since the long evacuation time can additionally reduce the laser ion source efficiency for the short-lived isotopes the throat diameter can be increased up to $1.5 \mathrm{~mm}$ to get this time about $190 \mathrm{~ms}$ for the $20 \mathrm{~mm}$ cell. The reduction factor of the gas cell efficiency due to radioactive decay is shown in Fig. 8 as a function of the isotope life time for the cells with $w=20 \mathrm{~mm}$ and $30 \mathrm{~mm}$ and throat diameters $d$ of $1 \mathrm{~mm}$ and $1.5 \mathrm{~mm}$. The smallest loss has the $20 \mathrm{~mm}$ cell with the $1.5 \mathrm{~mm}$ throat.

All components of the gas cell are made under the ultra high vacuum (UHV) standard. Parts of the cell are made of stainless steel, electropolished and are sealed by aluminum o-rings. The windows for the transverse laser entrance are sealed by silvercoated c-rings. The longitudinal entrance window is a fused silica viewport on a CF flange. The gas cell can be baked up to $200^{\circ} \mathrm{C}$ by heating elements located in the body of the cell.

The gas purity is a key issue for the performance of the laser ion source [5]. The gas handling system supplies argon or helium gas towards the separator frontend via two separate lines. The first one provides the gas to the main cell described above. Highpurity argon or helium (grade 6.0, 99.9999\%) from Air Products is additionally purified in a getter-based purifier (MonoTorr Phase II 3000 , SAES Pure Gas, Inc) down to the level below 1 ppb. Electropolished tubes that can be backed up to $200^{\circ} \mathrm{C}$ are used. The gas flow is regulated by a digital flow controller GF 120CXXC (Brooks Instrument) with a maximum rate of $15 \mathrm{sccm}$. In the second line no purification is needed, it supplies the gas to a different gas cell used only to visualize the flow pattern from de Laval nozzles by means of the Planar Laser Induced Fluorescence (PLIF) technique (see Section 2.3.3). In this cell the pressure conditions are similar but no purification is provided. Unlike in the main gas cell, acetone vapor is admixed to the noble gas. In this line the gas flow from the bottle is split in two channels; one going directly to the cell and the other passes through the container with acetone vapors. These two channels merge later on the way to the gas cell. The acetone concentration in the cell is regulated by flow controllers in both channels.

\subsubsection{Jet visualization and jet characterization}

The spectral resolution that can be achieved using the in-gas-jet ionization technique is mainly defined by the Mach number of the jet and its divergence. Studies of jets produced by nozzles with different Mach numbers will be carried out by PLIF of acetone molecules seeded into the gas. The visualization is realized by irradiation of the jet in the maximum of the absorption spectrum around $280 \mathrm{~nm}$ and by monitoring the fluorescence- and the phosphorescence emission by a CCD camera in a spectral range 

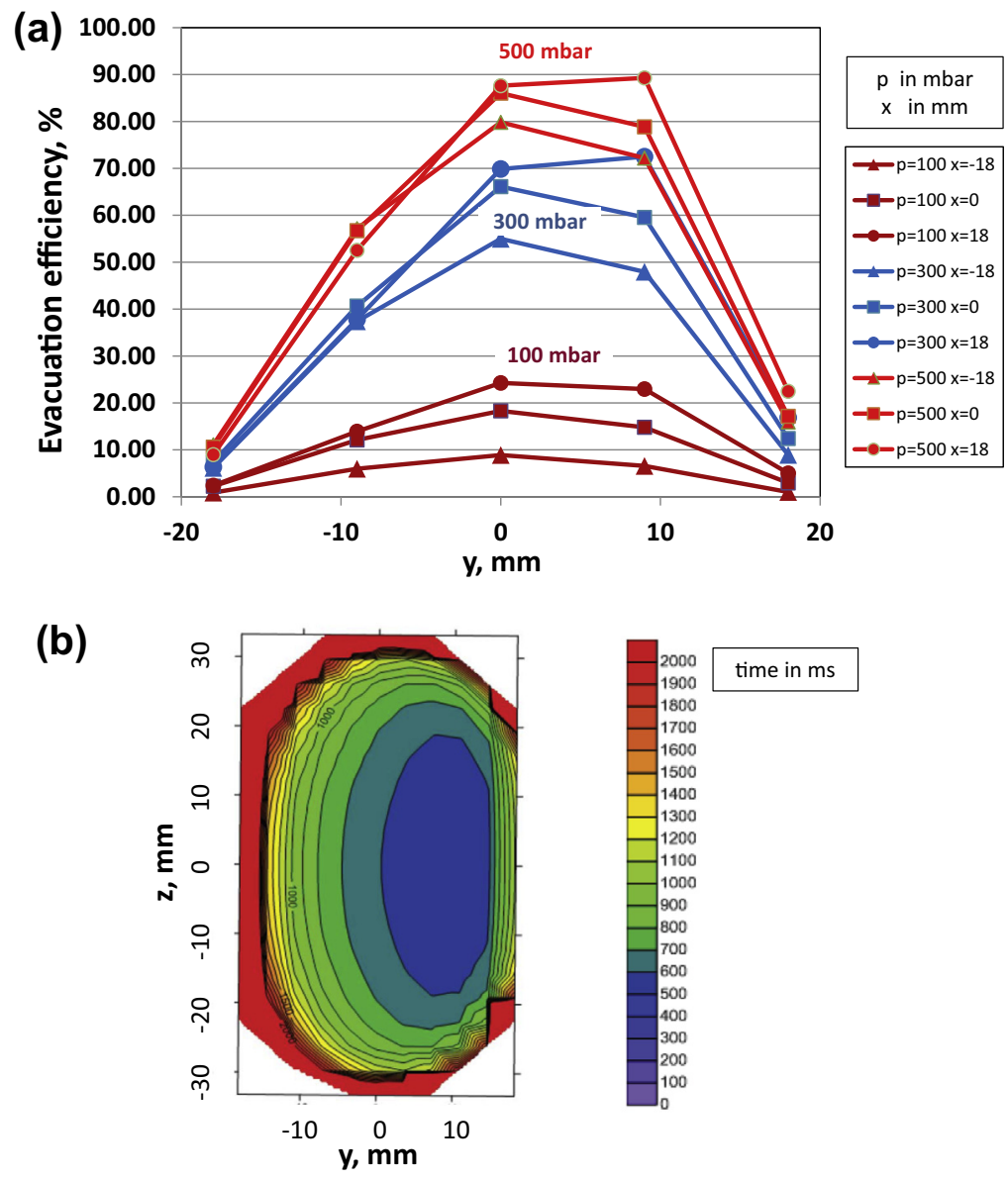

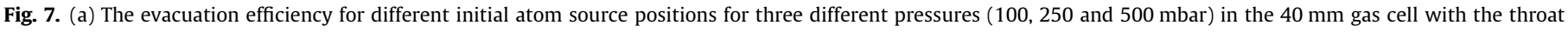
diameter of $1 \mathrm{~mm}$, (b) the evacuation time for different initial positions of atoms in the $40 \mathrm{~mm}$ gas cell; the $z$ axis is perpendicular to the $x-y$ plane in Fig. 6 a.

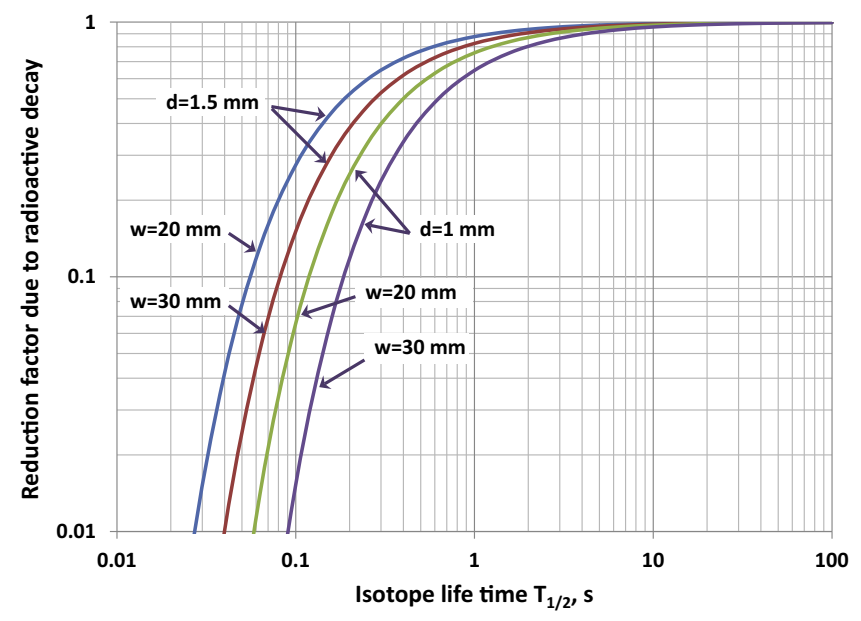

Fig. 8. Reduction factor of the gas cell efficiency due to radioactive decay for a $20 \mathrm{~mm}$ and a $30 \mathrm{~mm}$ gas cell evacuated through a nozzle with a throat diameter of $1 \mathrm{~mm}$ and $1.5 \mathrm{~mm}$.

of $400-550 \mathrm{~nm}$ [24]. In this technique a laser beam shaped in the form of a sheet of $50 \mathrm{~mm}$ in transverse (perpendicular to the jet) direction is used in the middle plane of the gas jet to excite the acetone molecules (see laser beam $\lambda_{2}$ in Fig. 3). The optimal background pressure in the gas cell chamber will be adjusted to obtain the minimal divergence of the jet.
After visual optimization of the flow field from different de Laval nozzles the PLIF technique will be used on atoms seeded in the jet. In this case a sheet of laser radiation will irradiate the gas jet in longitudinal or in transverse directions and the emitted fluorescent photons will be observed by an Intensified CCD camera (ICCD); see the side view in Fig. 3. The scattered laser light from the optical components is eliminated by optical filters and/or by a time gating of the ICCD. For the first tests copper atoms have been chosen. The atoms from the ground state $3 d^{10} 4 s^{2} S_{1 / 2}$ will be excited at $327.395 \mathrm{~nm}$ to the $3 \mathrm{~d}^{10} 4 \mathrm{p}^{2} \mathrm{P}_{1 / 2}^{0}$ state. The temperature, velocity and density of the atoms in the jet will be investigated by scanning the narrow band laser radiation across the atomic resonance and by monitoring the fluorescence at $578.213 \mathrm{~nm}$ which has a branching ratio of $0.2 \%$. The jet parameters will be deduced from the Doppler shifts and from the shape of the resonance in different parts of the jet.

\section{Conclusions and outlook}

The new IGLIS laboratory that is commissioned at KU Leuven allows to investigate selective laser ionization of atoms in both the gas cell and in the supersonic gas jet. Calculations indicate that a spectral resolution below $100 \mathrm{MHz}$ should be achievable in ingas-jet laser spectroscopy studies. This will allow measurements of the velocity distribution of seeded atoms in the cold environment of the jet produced by a de Laval nozzle. Simulations have been performed and optimum gas cell and nozzle designs are finalized. These experiments should allow to further investigate the 
gas-jet formation also in the presence of the S-RFQ ion guide and to characterize the different processes that will become important for laser spectroscopy of on-line produced radioactive isotopes:

- Because of the different masses of the carrier gas and the seeded atoms, neighboring isotopes can be differently accelerated and can attain different final velocities. This, so called, slipping effect can be larger for helium than for argon because of the larger mass difference. This effect should be studied to exclude systematic error because of possible Doppler shift of resonances.

- Formation of clusters during gas expansion in the de Laval nozzle can influence the efficiency of the ion source since radioactive atoms can form adducts with these clusters and will not undergo laser ionization. This effect will be studied by comparing the source efficiency of the Mach 3 nozzle with argon in which no clustering is possible with the Mach 8 nozzle. The other comparison will be made by using the Mach 8 nozzle but with helium as a carrier gas, which also excludes the formation of clusters.

- The spectral width of the atomic transition in the case of twostep laser ionization depends crucially on time delay between the first- and second step pulses. In particular the spectral line obtained with the delayed second step excitation exhibits no power broadening [25]. This phenomenon can be investigated by ionization in a supersonic gas jet where the Doppler contribution to the line width for heavy atoms is comparable with the laser bandwidth (see Fig. 9c in [12]). The frequency scan of the ion signal from the ionization region, which is spatially very well defined by overlapping of collimated laser beams and the gas jet at a $90^{\circ}$ geometry, can directly be compared with the one obtained in the PLIF measurement from the same part of the gas jet.

- The in-gas-jet ion source efficiency depends on the spatial overlapping of the continuous jet with high-repetition rate laser pulses. To exclude the duty-cycle losses the jet length should be at least $5.6 \mathrm{~cm}$ in case of argon and $17.7 \mathrm{~cm}$ in case of helium. The precise design of small scale de Laval nozzles and accurate adjustments of the background pressures in the gas cell chamber are required to produce supersonic jets of such lengths.

Finally, the gas cell-nozzle combination will be optimized for various studies of radioactive isotopes produced in different types of nuclear reactions and in different regions of the nuclear chart where REGLIS will be put in work.

\section{Acknowledgments}

This work was supported by FWO-Vlaanderen (Belgium), by GOA/2010/010 (BOF KU Leuven), by the IAP Belgian Science Policy
(BriX network P7/12), by a Grant from the European Research Council (ERC-2011- AdG-291561-HELIOS) and by a Ph.D grant of the Agency for Invasion by Science and Technology (IWT).

\section{References}

[1] Yu.A. Kudryavtsev, J. Andrzejewski, N. Bijnens, S. Franchoo, J. Gentens, M Huyse, A. Piechaczek, I. Reusen, J. Szerypo, P. Van den Bergh, P. Van Duppen, L. Vermeeren, J. Wauters, A. Wöhr, Nucl. Instr. Meth. Phys. Res. B 114 (1996) 350

[2] M. Huyse, M. Facina, Yu. Kudryavtsev, P. Van Duppen, Nucl. Instr. Meth. B 187 (2002) 535.

[3] Yu. Kudryavtsev, M. Facina, M. Huyse, J. Gentens, P. Van den Bergh, P. Van Duppen, Nucl. Instr. Meth. B 204 (2003) 336.

[4] M. Facina, B. Bruyneel, S. Dean, J. Gentens, M. Huyse, Yu. Kudryavtsev, P. Van den Bergh, P. Van Duppen, Nucl. Instr. Meth. B 226 (2004) 401.

[5] Yu. Kudryavtsev, B. Bruyneel, M. Huyse, J. Gentens, P. Van den Bergh, P. Van Duppen, L. Vermeeren, Nucl. Instr. Meth. B 179 (2001) 412.

[6] Yu. Kudryavtsev, R. Ferrer, M. Huyse, P. Van den Bergh, P. Van Duppen, L. Vermeeren, Rev. Sci. Instrum. 85 (2014) 02 B915.

[7] Yu. Kudryavtsev, T. Cocolios, J. Gentens, M. Huyse, O. Ivanov, D. Pauwels, T. Sonoda, P. Van den Bergh, P. Van Duppen, Nucl. Instr. Meth. B 267 (2009) 2908

[8] T.E. Cocolios, A.N. Andreyev, B. Bastin, N. Bree, J. Buscher, J. Elseviers, J. Gentens, M. Huyse, Yu. Kudryavtsev, D. Pauwels, T. Sonoda, P. Van den Bergh, P. Van Duppen, Phys. Rev. Lett. 103 (2009) 102501.

[9] R. Ferrer, N. Bree, T.E. Cocolios, I.G. Darby, H. De Witte, W. Dexters, J. Diriken, J. Elseviers, S. Franchoo, M. Huyse, N. Kesteloot, Yu. Kudryavtsev, D. Pauwels, D. Radulov, T. Roger, H. Savajols, P. Van Duppen, M. Venhart, Phys. Lett. B 728 (2014) 191.

[10] I.D. Moore, J. Billowes, P. Campbell, T. Eronen, C. Geppert, A. Jokinen, P. Karvonen, T. Kessler, B. Marsh, A. Nieminen, H. Penttilä, S. Rinta-Antila, T. Sonoda, B. Tordoff, K. Wendt, J. Äysto, AIP Conf. Proc. 831 (2006) 511.

[11] T. Sonoda, T. Cocolios, J. Gentens, M. Huyse, O. Ivanov, Yu. Kudryavtsev, D. Pauwels, P. Van den Bergh, P. Van Duppen, Nucl. Instr. Meth. B 267 (2009) 2918.

[12] Yu. Kudryavtsev, R. Ferrer, M. Huyse, P. Van den Bergh, P. Van Duppen, Nucl Instr. Meth. B 297 (2013) 7.

[13] S. Raeder et al., Proceedings of this Conference, Nucl. Instr. Meth. B 376 (2016) 382.

[14] Yu. Kudryavtsev, Hyperfine Interact. 74 (1992) 171.

[15] K.T. Flanagan, J. Billowes, M.L. Bissell, I. Budinčević, T.E. Cocolios, R.P. de Groote, S. De Schepper, V.N. Fedosseev, S. Franchoo, R.F. García Ruiz, H. Heylen, K.M. Lynch, B.A. Marsh, G. Neyens, T.J. Procter, R.E. Rossel, S. Rothe, I. Strashnov, H.H. Stroke, K.D.A. Wendt, Hyperfine Interact. 227 (2014) 131.

[16] R. Ferrer, B. Bastin, D. Boilley, P. Creemers, P. Delahaye, E. Lienard, X. Flechard, S. Franchoo, L. Ghys, M. Huyse, Yu. Kudryavtsev, N. Lecesne, H. Lu, F. Lutton, E. Mogilevskiy, D. Pauwels, J. Piot, D. Radulov, L. Rens, H. Savajols, J.C. Thomas, E. Traykov, C. Van Beveren, P. Van den Bergh, P. Van Duppen, Nucl. Instr. Meth. B 317 (2013) 570 .

[17] F. Déchery, A. Drouart, H. Savajols, J. Nolen, M. Authier, A.M. Amthor, D. Boutin, O. Delferriére, B. Gall, A. Hue, B. Laune, F. Le Blanc, S. Manikonda, J. Payet, M.-H. Stodel, E. Traykov, D. Uriot, Eur. Phys. J. A 51 (2015) 66.

[18] <http://research.kek.jp/group/kekrnb/iglis-net/index.html>.

[19] G. Dupeyrat, J.B. Marquette, B.R. Rowe, Phys. Fluids 28 (1985) 1273.

[20] D.B. Atkinson, M.A. Smith, Rev. Sci. Instrum. 66 (1995) 4434.

[21] G. Bollen, S. Schwarz, D. Davies, P. Lofy, D. Morrissey, R. Ringle, P. Schury, T. Sun, L. Weissman, Nucl. Instr. Meth. A 532 (2004) 203.

[22] <http://www.comsol.com/>.

[23] J.F. Ziegler, J.P Biersack, <www.srim.org>.

[24] A. Lozano, B. Yip, R.K. Hanson, Exp. Fluids 13 (1992) 369.

[25] N. Vitanov, B. Shore, L. Yatsenko, K. Böhmer, T. Halfmann, T. Rickes, K. Bergmann, Opt. Commun. 199 (2001) 117. 